\title{
Oxaliplatin promotes siMAD2L2-induced apoptosis in colon cancer cells
}

\author{
LU MA ${ }^{1,2^{*}}$, XIN LI $^{1,2^{*}}$, XIAOPENG ZHAO ${ }^{1,2}$, HAOTONG SUN ${ }^{1,2}$, \\ FEIFEI KONG ${ }^{3}$, YUANJIE $\mathrm{LI}^{1,2}$, YU SUI ${ }^{1,2}$ and FANG XU ${ }^{1,2}$ \\ ${ }^{1}$ Department of Medical Genetics and Cell Biology, School of Basic Medical Sciences; \\ ${ }^{2}$ Key Laboratory of Reproduction and Genetics, Ningxia Medical University, Yinchuan, Ningxia 750004; \\ ${ }^{3}$ Department of Oncology, Qufu People's Hospital, Qufu, Shandong 273100, P.R. China
}

Received September 15, 2020; Accepted May 26, 2021

DOI: $10.3892 / \mathrm{mmr} .2021 .12268$

\begin{abstract}
The clinical efficacy of colorectal tumor treatment is restricted due to platinum agent resistance. Translesion DNA synthesis (TLS) has been shown to contribute to this resistance; however, the exact molecular mechanism remains unknown. The present study aimed to investigate the possible function of the core of the TLS polymerase mitotic arrest deficient 2 like 2 (MAD2L2) in drug sensitivity, in order to provide a treatment rationale for platinum-based chemotherapy in colon cancer. In the present study, MAD2L2 was knocked down using MAD2L2-specific small interfering (si)RNA. HCT116 and SW620 cells were treated with oxaliplatin and MG132; oxaliplatin is a platinum compound that induces DNA damage and MG132 is a potent proteasome inhibitor. Cell viability was determined using an MTT assay. Cell apoptosis was examined via flow cytometry and TUNEL assay. The activity of proteasome 26S subunit, non-ATPase 13 (PSMD13) was detected using ELISA, while the expression levels of apoptotic-related proteins were detected via western blotting. The results demonstrated that cells treated with oxaliplatin or MG132 alone had decreased viability, but a synergistic effect was not observed after co-treatment. In addition, the knockdown of MAD2L2 caused by siMAD2L2 or oxaliplatin treatment increased the expression levels of the pro-apoptotic proteins Bax and Bak and decreased the expression levels of the anti-apoptotic protein Bcl-2, compared with the negative control group. Moreover, MG132 alleviated the decrease in MAD2L2 expression, while reducing siMAD2L2-induced cell apoptosis. These
\end{abstract}

Correspondence to: Professor Fang Xu, Department of Medical Genetics and Cell Biology, School of Basic Medical Sciences, Ningxia Medical University, 1160 Shengli Street, Xingqing, Yinchuan, Ningxia 750004, P.R. China

E-mail: xufang@nxmu.edu.cn

${ }^{*}$ Contributed equally

Key words: mitotic arrest deficient 2 like 2, oxaliplatin, colon cancer cell, ubiquitin-proteasome pathway, cell apoptosis results indicate that oxaliplatin promotes siMAD2L2-induced apoptosis in colon cancer cells. This process was associated with the Bcl-2 and ubiquitin-proteasome pathway. Overall, the present study provides a theoretical basis for improving the clinical efficacy of colon cancer by combining chemotherapy and gene therapy.

\section{Introduction}

Globally, colorectal cancer (CRC) is the third most common form of cancer, and is the second most common cause of cancer-associated mortality $(1,2)$. Although CRC is considered to be mainly prevalent in developed countries, the incidence is also rising rapidly in developing countries $(3,4)$. Further basic and clinical research would undoubtedly accelerate the progress in the treatment of CRC.

Oxaliplatin has been proven for its specificity against colorectal tumors, thereby becoming a standard therapeutic in the management of this malignancy (5). However, its clinical application is restricted due to drug resistance and toxic side effects (6). Although research has been conducted through laboratory-based and clinical studies to improve and optimize therapeutic potency, high efficiency and safe treatment options remain the focus of ongoing studies. In recent years, chemotherapeutic drugs and gene-based combination therapy have become one of the most promising and active research fields in medicine (7).

Oxaliplatin functions predominantly via the formation of drug-DNA adducts that block DNA synthesis, thereby triggering a cellular response and eventually leading to cell apoptosis (8). Translesion DNA synthesis (TLS) is a strategy for tolerating DNA damage, which serves an essential role in the maintenance of genome stability (9). Recent studies have revealed that TLS polymerase contributes to the development of platinum resistance in cancer cells (10), particularly polymerase $\zeta$ (11). REV3, the catalytic subunit of polymerase $\zeta$, has attracted increased attention from researchers and a series of related reports can be found (12-15), while another subunit, mitotic arrest deficient 2 like 2 (MAD2L2; also known as REV7), has been rarely studied (11).

The ubiquitin (Ub) proteasome pathway (UPP) is the most important pathway involved in intracellular protein 
degradation (16). The $26 \mathrm{~S}$ proteasome is an essential multi-catalytic protease complex, which serves key roles in the function of the UPP. The 26S proteasome consists of proteasome 26S subunit, non-ATPase 13 (PSMD13) and a few additional components, such as proteasomal Ub receptor, proteasome 26S subunit, ATPase 1, Ub specific peptidase 14 and Ub C-terminal hydrolases 37 (17,18). Specific proteins involved in DNA damage repair, cell cycle regulation and apoptosis are the targets of the UPP (19). However, the underlying regulatory mechanism between the expression level of MAD2L2 and the UPP has not yet been elucidated. The current study proposed that the UPP may be involved in DNA damage repair of tumor cells caused by TLS and oxaliplatin, which may be mediated by regulating the expression levels of MAD2L2, thereby affecting cell apoptosis. MG132, a specific inhibitor of the $26 \mathrm{~S}$ proteasome, was selected as an appropriate experimental compound to be used in the present study.

The present study aimed to investigate the antitumor effect and possible mechanism of MAD2L2, in order to provide a rationale for the clinical treatment of colon cancer.

\section{Materials and methods}

Cell culture and treatment. Colon cancer cell lines, HCT116 and SW620, were obtained from the American Type Culture Collection. Cells were incubated in DMEM (Gibco; Thermo Fisher Scientific, Inc.) supplemented with 10\% FBS (Gibco; Thermo Fisher Scientific, Inc.) and Penicillin-Streptomycin Solution (10,000 U/ml; Beyotime Institute of Biotechnology) at $37^{\circ} \mathrm{C}$ and $5 \% \mathrm{CO}_{2}$.

Gene expression was knocked down using small interfering (si)RNA. Cells were transfected with $10 \mu \mathrm{M}$ MAD2L2 siRNA and negative control siRNA using Lipofectamine ${ }^{\circledR}$ RNAi MAX (Invitrogen; Thermo Fisher Scientific, Inc.) at $37^{\circ} \mathrm{C}$ for $48 \mathrm{~h}$. siRNA were designed and synthesized by Shanghai GenePharma Co., Ltd. The siRNA sequences were as follows: MAD2L2 forward, 5'-AAGAUGCAGCUUUAC GUGGAATT-3' and reverse, 5'-UUCCACGUAAAGCUG CAUCUUTT-3'; and negative control forward, 5'-UUCUCC GAACGUGUCACGUTT-3' and reverse, 5'-ACGUGACAC GUUCGGAGAATT-3'. Reverse transcription-quantitative PCR (RT-qPCR) and western blotting were performed to identify transfection efficiency after 48-h transfection.

Cells were treated with oxaliplatin(Jiangsu Hengrui Medicine Co., Ltd.) and MG132 (MedChemExpress) at $37^{\circ} \mathrm{C}$ for $24 \mathrm{~h}$. The concentrations of both drugs under the current experimental condition were recalculated according to previous studies: Oxaliplatin, $50 \mu \mathrm{M}$ in HCT116 cells and $90 \mu \mathrm{M}$ in SW620 cells; and MG132, $18 \mu \mathrm{M}$ in HCT116 cells and $36 \mu \mathrm{M}$ in SW620 cells. As a control, the blank group received the same volume of $1 \%$ dimethyl sulfoxide (DMSO) vehicle as the other groups.

$R T$ - $q P C R$. RT-qPCR was performed to detect and quantify mRNA. Total RNA was extracted from experimental cells using TRIzol ${ }^{\circledR}$ reagent (Beyotime Institute of Biotechnology). cDNA was produced using SYBR Premix Ex TaqII (TliRNaseH Plus; Takara Biotechnology Co., Ltd.) from the extracted RNA. RT reactions were conducted under the following conditions: $37^{\circ} \mathrm{C}$ for $15 \mathrm{~min}, 85^{\circ} \mathrm{C} 5 \mathrm{sec}$, maintained at $4^{\circ} \mathrm{C}$. qPCR amplification reactions were carried out in the Applied Biosystems ${ }^{\mathrm{TM}} 7500$ Fast Real Time PCR system using a PowerUp ${ }^{\mathrm{TM}}$ SYBR ${ }^{\mathrm{TM}}$ Green Master Mix (both Thermo Fisher Scientific, Inc.). Available primers were obtained from Sangon Biotech, Co., Ltd., and the sequences of primers are as follows: MAD2L2 forward, 5'-CCAGGCTGTACCTTCACA GTC-3' and reverse, 5'-TCTTCCACGTAAAGCTGCATC-3'; and GAPDH forward, 5'-ACCCACTCCTCCACCTTTGAC-3' and reverse, 5'-CACCACCCTGTTGCTGTAGCC-3'. The thermocycling conditions were as follows: Initial denaturation at $95^{\circ} \mathrm{C}$ for $2 \mathrm{~min}$; followed by 40 cycles of denaturation at $95^{\circ} \mathrm{C}$ for $3 \mathrm{sec}$ and annealing/elongation at $60^{\circ} \mathrm{C}$ for $30 \mathrm{sec}$; followed by a final extension step at $60^{\circ} \mathrm{C}$ for $1 \mathrm{~min}$. Finally, the relative gene expression values were calculated using the $2^{-\Delta \Delta C \mathrm{q}}$ method (20).

Western blotting. Western blotting was performed to separate and identify proteins. Total proteins were extracted using RIPA buffer (Beyotime Institute of Biotechnology), followed by the determination of the protein concentration using a BCA kit (Nanjing KeyGen Biotech. Co., Ltd.). Proteins (20-30 $\mu \mathrm{g} /$ well) were separated via $12 \%$ SDS-PAGE at $120 \mathrm{~V}$ and then transferred into $0.2-\mu \mathrm{m}$ PVDF membrane under wet conditions at $300 \mathrm{~mA}$. The blotted membranes were blocked with $5 \%$ non-fat milk for $2 \mathrm{~h}$ at room temperature. Antibodies were diluted in TBS-Tween-20 (1\% TBS and $0.1 \%$ Tween). Primary antibodies were incubated at $4^{\circ} \mathrm{C}$ overnight and secondary antibodies were incubated at room temperature for $1 \mathrm{~h}$. Chemiluminescent signals were detected using Pierce $^{\mathrm{TM}}$ ECL Western Blotting substrate (cat. no. 32109; Thermo Fisher Scientific, Inc.). Images were captured using Bio-Rad ChemiDOC XRS ${ }^{+}$system (Bio-Rad Laboratories, Inc.) and analyzed by Image Lab Software (version 5.2.1; Bio-Rad Laboratories, Inc.). The following antibodies were used: Primary antibodies, MAD2L2 (cat. no. sc135977; 1:500; Santa Cruz Biotechnology, Inc.), PSMD13 (cat. no. ab229812; 1:1,000; Abcam), Bax (cat. no. bs-0127R; 1:200; BIOSS), Bak (cat. no. bs-1284R; 1:200; BIOSS), Bcl-2 (cat. no. bs-0032R; 1:200; BIOSS) and GAPDH (cat. no. AB-P-R 001; 1:1,000; Hangzhou Goodhere Biotechnology Co., Ltd.); Secondary antibodies, goat Anti-Rabbit IgG H\&L (HRP) (cat. no. ab6721; 1:10,000; Abcam) and goat Anti-Mouse IgG H\&L (HRP) (cat. no. ab205719; 1:10,000; Abcam).

MTT assay. Cell viability was investigated using an MTT assay kit (Nanjing KeyGen Biotech Co., Ltd.). The cell count was adjusted to $1 \times 10^{4}$ cells $/ \mathrm{ml}$. Cells were treated with oxaliplatin and MG132 in 96-well plate at $37^{\circ} \mathrm{C}$ for $24 \mathrm{~h}$. MTT solution was added and incubated for $30 \mathrm{~min}$ at $37^{\circ} \mathrm{C}$ until the solution turned purple. DMSO was used for dissolving the purple crystals. Absorbance was measured using Multiskan ${ }^{\mathrm{TM}} \mathrm{GO}$ microplate spectrophotometer (Thermo Fisher Scientific, Inc.) at $570 \mathrm{~nm}$. The percentage of cell viability was calculated.

Flow cytometry. Cell apoptosis was detected via flow cytometry (BD Accuri ${ }^{\mathrm{TM}}$ C6; BD Biosciences). EDTA-free trypsin (Hyclone Laboratories, Inc.) was used to detach the experimental cells. According to the instructions of the Annexin V-FITC/PI kit (BD Biosciences), each group of cells was incubated with $5 \mu \mathrm{l}$ Annexin V-FITC for $15 \mathrm{~min}$ at $2-8^{\circ} \mathrm{C}$. 
Table I. Effects of oxaliplatin and/or MG132 on the viability of human colorectal cancer cells.

\begin{tabular}{lcccc}
\hline Cells & Blank & Oxaliplatin & MG132 & Oxaliplatin and MG132 \\
\hline HCT116 & $0.904 \pm 0.001$ & $0.656 \pm 0.043^{\mathrm{a}}$ & $0.257 \pm 0.011^{\mathrm{a}}$ & $0.351 \pm 0.005^{\mathrm{a}}$ \\
SW620 & $0.909 \pm 0.0002$ & $0.665 \pm 0.011^{\mathrm{a}}$ & $0.215 \pm 0.006^{\mathrm{a}}$ & $0.258 \pm 0.007^{\mathrm{a}}$ \\
\hline
\end{tabular}

${ }^{a} \mathrm{P}<0.001$ vs. blank group. The data are presented as the mean $\pm \mathrm{SD} . \mathrm{n}=3$. The cells were treated with oxaliplatin or MG132 alone, or co-treated with oxaliplatin and MG132 for $24 \mathrm{~h}$ (oxaliplatin was administered at 50 and $90 \mu \mathrm{M}$ to HCT116 and SW620 cells, respectively; MG132 was administered at 18 and $36 \mu \mathrm{M}$ to HCT116 and SW620 cells, respectively). Cell viability was assessed using the MTT assay. One-way ANOVA followed by Tukey's post-hoc test was used to determine the statistical significance of the differences between the groups.

A
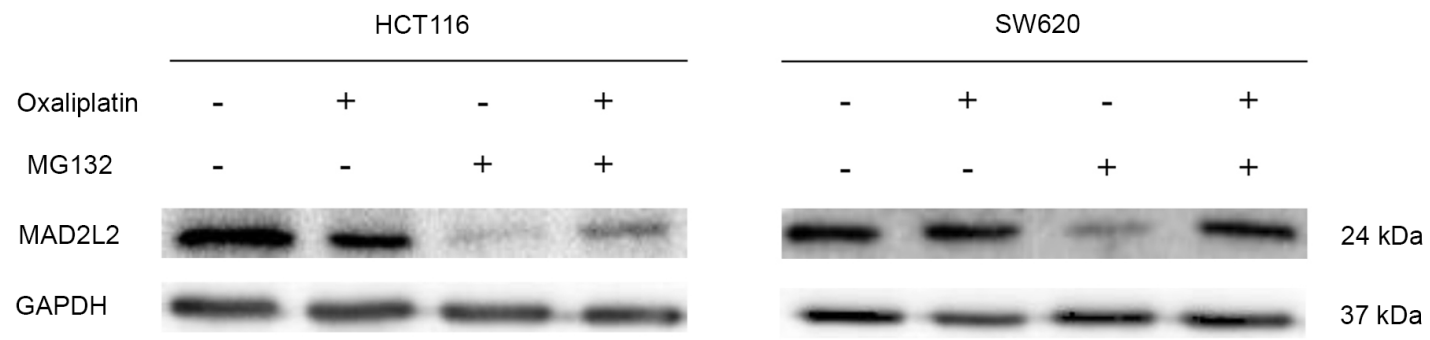

B
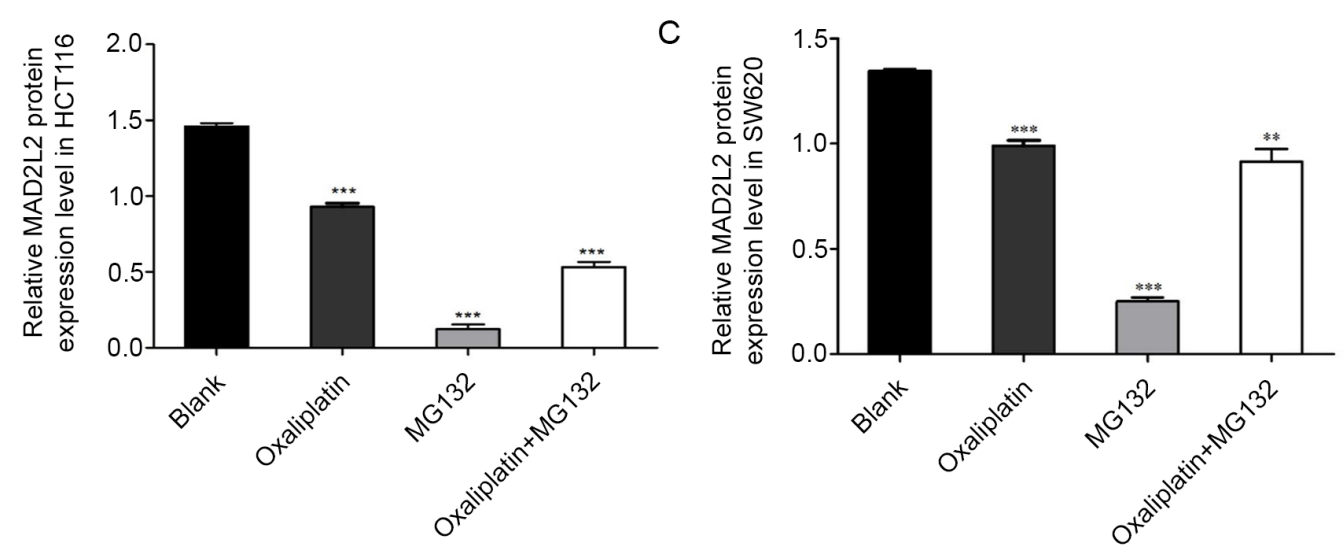

Figure 1. MAD2L2 expression is downregulated by oxaliplatin and MG132 in human colon cancer cells. (A) Western blotting was performed to detect the protein expression levels of MAD2L2 in HCT116 and SW620 cells. The cells were treated with oxaliplatin or MG132 alone, or co-treated with oxaliplatin + MG132 for $24 \mathrm{~h}$ (oxaliplatin was incubated at 50 and $90 \mu \mathrm{M}$ with HCT116 and SW620 cells, respectively; MG132 was incubated at 18 and $36 \mu \mathrm{M}$ with HCT116 and SW620 cells, respectively). One-way ANOVA followed by Tukey's multiple comparisons test was used to determine the statistical significance in (B) HCT116 and (C) SW620 cells. The data are presented as mean \pm SD. $n=3 .{ }^{* *} \mathrm{P}<0.01,{ }^{* * *} \mathrm{P}<0.001$ vs. blank. MAD2L2, mitotic arrest deficient 2 like 2.

Subsequently, $10 \mu \mathrm{l}$ PI was added and incubated for $5 \mathrm{~min}$ at $2-8^{\circ} \mathrm{C}$. Cells were analyzed within $30 \mathrm{~min}$ after staining using BD Accuri C6 software (version 5.0; BD Biosciences). The apoptotic rate was calculated as the percentage of early and late apoptotic cells.

TUNEL assay. DNA fragmentation, which is a marker of cell apoptosis (2), was detected using the TUNEL BrightGreen Apoptosis Detection kit (cat. no. A112-02; Vazyme Biotech Co., Ltd.). The cells were cultured on microscope slides, fixed with $4 \%$ formaldehyde at room temperature for $15 \mathrm{~min}$ and permeabilized with $0.25 \%$ Triton ${ }^{\circledR} \mathrm{X}-100$ at room temperature for $20 \mathrm{~min}$. TUNEL staining was performed according to the manufacturer's instructions; cells were stained with $50 \mu \mathrm{l}$ Recombinant TdT Enzyme at $37^{\circ} \mathrm{C}$ for $60 \mathrm{~min}$ and with $50 \mu \mathrm{l}$ BrightGreen Labeling Mix at $37^{\circ} \mathrm{C}$ for $60 \mathrm{~min}$. The slides were then immersed into $2 \mu \mathrm{g} / \mathrm{ml}$ DAPI solution and stained in the dark for $5 \mathrm{~min}$. The samples were examined via fluorescence microscopy (DFC450 C; Leica Microsystems, Inc.); 10 visual fields were randomly selected per slide at x400 magnification.

ELISA. Total cell protein was extracted using RIPA buffer (Beyotime Institute of Biotechnology) from experimental cells. The activity of PSMD13 was detected using an ELISA kit (cat. no. ml-55255; Enzyme-linked Biotechnology Co., Ltd.). According to the manufacturer's instructions, standard wells, testing sample wells and blank wells were set. Standard and blank proteins were obtained from the ELISA kit. Standard protein was diluted to a range of 0, 50, 100, 150, 200, 250 and $300 \mathrm{ng} / \mathrm{ml}$. Each concentration of these standard samples was added to standard wells, $50 \mu \mathrm{l} /$ well; extracted protein samples were added to the testing well, $50 \mu \mathrm{l} /$ well; And $50 \mu \mathrm{l}$ blank protein was added to the blank well. Subsequently, $80 \mu \mathrm{l}$ HRP-conjugated antibody was immediately added to the 
Table II. Effects of siMAD2L2 in combination with oxaliplatin and/or MG132 on the viability of human colorectal cancer cells.

\begin{tabular}{lll}
\hline Treatment & HCT116 & SW620 \\
\hline Negative & $0.905 \pm 0.009$ & $0.942 \pm 0.013$ \\
siMAD2L2 & $0.573 \pm 0.013^{\mathrm{a}}$ & $0.539 \pm 0.002^{\mathrm{a}}$ \\
siMAD2L2 and oxaliplatin & $0.270 \pm 0.005^{\mathrm{a}, \mathrm{b}}$ & $0.191 \pm 0.004^{\mathrm{a}, \mathrm{b}}$ \\
siMAD2L2 and MG132 & $0.676 \pm 0.008^{\mathrm{a}-\mathrm{c}}$ & $0.666 \pm 0.006^{\mathrm{a}-\mathrm{c}}$ \\
siMAD2L2, oxaliplatin and MG132 & $0.363 \pm 0.009^{\mathrm{a}-\mathrm{d}}$ & $0.377 \pm 0.0004^{\mathrm{a}-\mathrm{d}}$ \\
\hline
\end{tabular}

${ }^{\mathrm{a}} \mathrm{P}<0.05$ vs. negative group; ${ }^{\mathrm{b}} \mathrm{P}<0.05$ vs. siMAD2L2 group; ${ }^{\mathrm{c}} \mathrm{P}<0.05$ vs. siMAD2L2 + oxaliplatin group; ${ }^{\mathrm{P}}<0.05$ vs. siMAD2L2 + MG132 group. The data are presented as the mean $\pm \mathrm{SD}$. $\mathrm{n}=3$. One-way ANOVA followed by Tukey's multiple comparisons test was used to determine statistical significance. The cells were treated with negative siRNA, siMAD2L2, siMAD2L2 + oxaliplatin, siMAD2L2 + MG132, siMAD2L2 + oxaliplatin + MG132 (oxaliplatin was incubated for $24 \mathrm{~h}$ at 50 and $90 \mu \mathrm{M}$ with HCT116 and SW620 cells, respectively; MG132 was incubated for $24 \mathrm{~h}$ at 18 and $36 \mu \mathrm{M}$ with HCT116 and SW620 cells, respectively). Cell viability was measured using the MTT assay. siRNA/si, small interfering RNA; MAD2L2, mitotic arrest deficient 2 like 2.

A

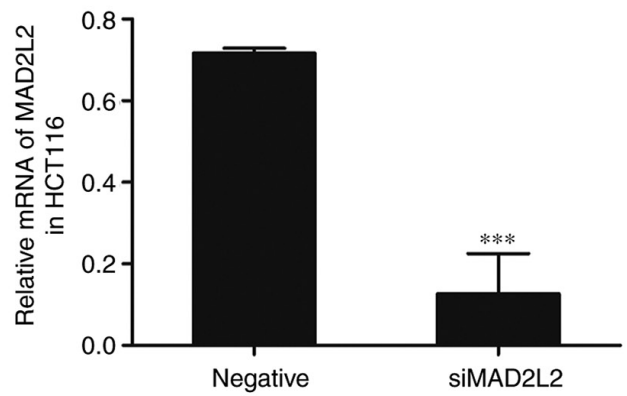

B

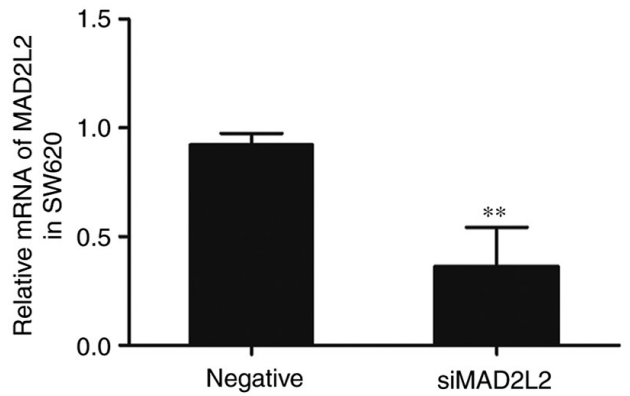

Figure 2. siMAD2L2 transfection downregulates the expression levels of MAD2L2 in human colon cancer cells. Negative siRNA and siMAD2L2 sequences were separately transfected into the cells, which were incubated for $24 \mathrm{~h}$. Reverse transcription-quantitative PCR analysis was performed to identify the transfection efficiency. The mRNA expression levels of MAD2L2 were assessed in (A) HCT116 and (B) SW620 cells. An unpaired Student's t-test was used to determine the statistical significance of the differences between the groups. The data are presented as the mean $\pm \mathrm{SD}$. $\mathrm{n}=3$. ${ }^{* * *} \mathrm{P}<0.01$, ${ }^{* * * *} \mathrm{P}<0.001 \mathrm{vS}$. negative. MAD2L2, mitotic arrest deficient 2 like 2; si, small interfering RNA.

wells and incubated at $37^{\circ} \mathrm{C}$ for $1 \mathrm{~h}$. Chromogenic substrate $\mathrm{A}$ (50 $\mu \mathrm{l})$ and chromogenic substrate $\mathrm{B}(50 \mu \mathrm{l})$ were added and incubated at $37^{\circ} \mathrm{C}$ for $10 \mathrm{~min}$, in the dark. Finally, $50 \mu \mathrm{l}$ stop solution were added to each well to terminate the reaction. The optical density was measured at $450 \mathrm{~nm}$. A standard curve was constructed and the corresponding concentration of PSMD13 was calculated.

Statistical analysis. SPSS 21.0 (IBM Corp.) was used for statistical analysis and GraphPad Prism 5 (GraphPad Software, Inc.) was used to generate the figures and mark the statistical difference. Data are presented as the mean \pm SD. An unpaired Student's t-test was used for comparison between two independent samples. One-way ANOVA was used for comparisons between groups, followed by Tukey's multiple comparisons test as the post-hoc test. $\mathrm{P}<0.05$ was considered to indicate a statistically significant difference. All experiments were repeated $\geq 3$ times.

\section{Results}

MAD2L2 is regulated by oxaliplatin andMG132 in human colon cancer cells. Cells were treated with oxaliplatin and MG132. The MTT data indicated that cells treated with oxaliplatin or MG132 had a significant decrease in their viability compared with the blank group, but a synergistic effect was not observed during the co-treatment of oxaliplatin and MG132 (Table I). The western blotting results demonstrated that oxaliplatin and MG132 caused a significant decrease in the protein expression level of MAD2L2, but a synergistic effect was not observed in the co-treatment group (Fig. 1). These results indicated that the expression level of MAD2L2 was decreased by oxaliplatin and MG132 in human colon cancer cells, but no synergistic effects were observed.

Knockdown of MAD2L2 promotes Bcl-2-mediated apoptosis of colon cancer cells. Since oxaliplatin and MG132 were identified to affect the expression level of MAD2L2, the associations between them were investigated. Cells were transfected with siMAD2L2; compared with the negative control group, the mRNA expression levels of MAD2L2 were significantly downregulated in the siMAD2L2 group (Fig. 2). Subsequently, the experimental cells were treated with oxaliplatin and MG132. The MTT data revealed that, compared with the negative control group, siMAD2L2 caused a significant decrease in cell viability (Table II). Compared with the siMAD2L2 group, cell viability was suppressed by the co-treatment of siMAD2L2 and oxaliplatin, whereas it was increased by the co-treatment of siMAD2L2 and MG132. A synergistic effect was not observed during triple co-treatment with siMAD2L2, oxaliplatin and MG132 (Table II). 
A
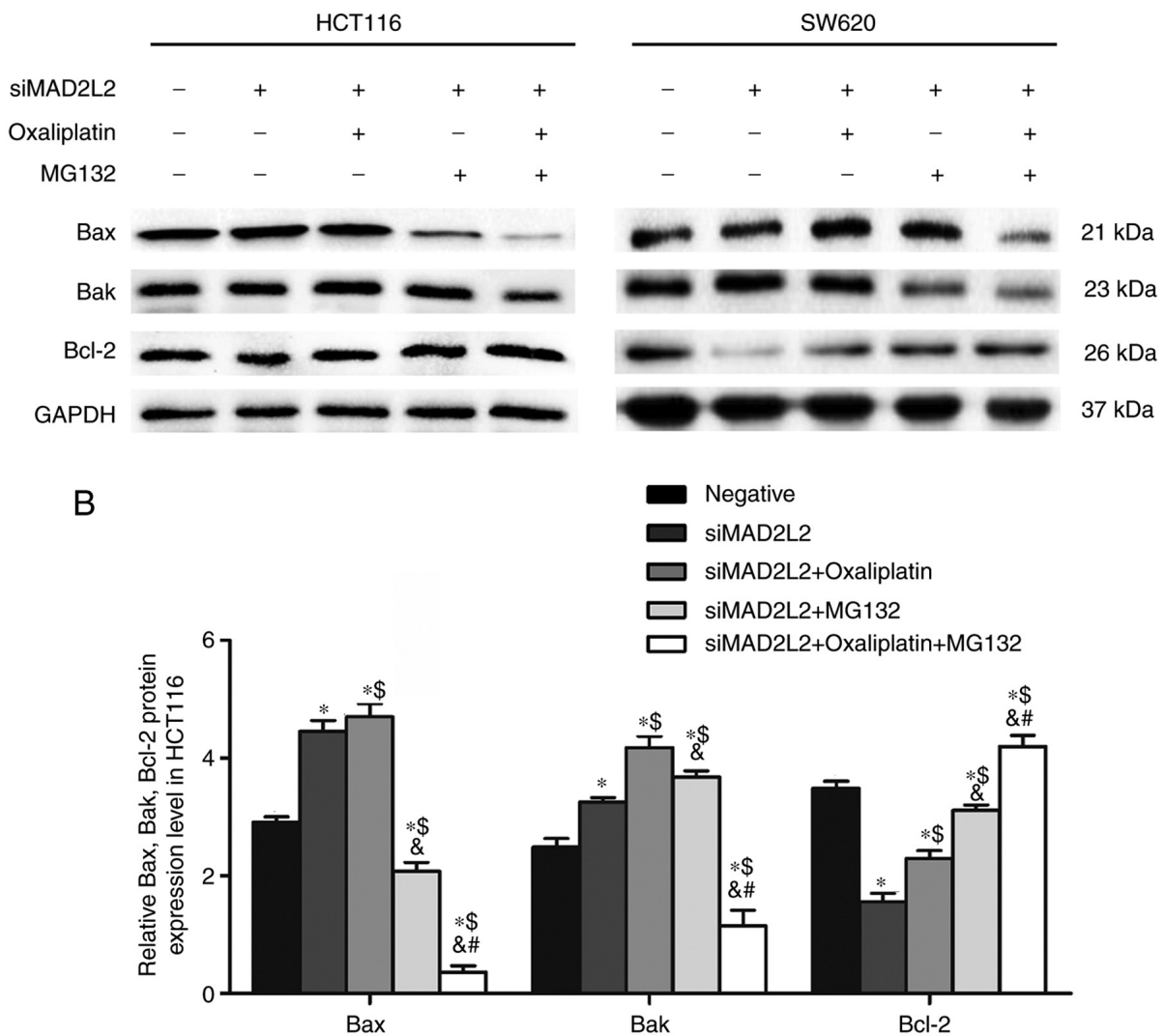

C

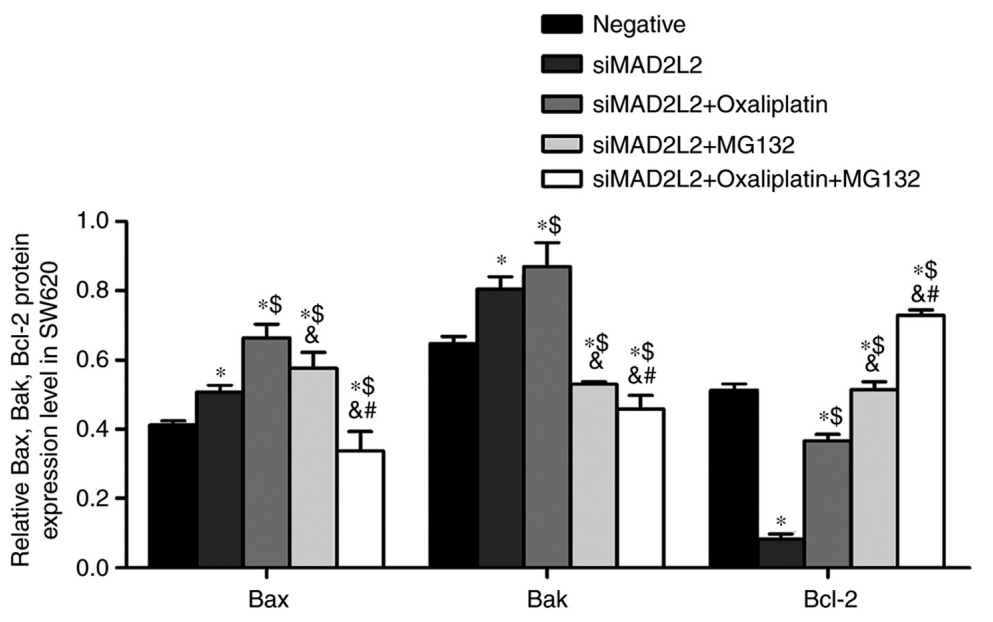

Figure 3. Knockdown of MAD2L2 expression promotes Bcl-2-mediated apoptosis of colon cancer cells. The cells were treated with negative siRNA, siMAD2L2, siMAD2L2 + oxaliplatin, siMAD2L2 + MG132 and siMAD2L2 + oxaliplatin + MG132 (oxaliplatin was incubated for $24 \mathrm{~h}$ at 50 and $90 \mu$ M with HCT116 and SW620 cells, respectively; MG132 was incubated for $24 \mathrm{~h}$ at 18 and $36 \mu \mathrm{M}$ with HCT116 and SW620 cells, respectively). Western blotting was performed to detect the expression levels of cell apoptosis-associated proteins. (A) Expression levels of Bax, Bak and Bcl-2 proteins in HCT116 and SW620 cells. One-way ANOVA followed by Tukey's multiple comparisons test was used to determine the statistical significance in (B) HCT116 and (C) SW620 cells. The data are presented as the mean \pm SD. $n=3$. ${ }^{*} \mathrm{P}<0.05$ vs. negative group; ${ }^{\$} \mathrm{P}<0.05$ vs. siMAD2L2 group; ${ }^{\circledR} \mathrm{P}<0.05$ vs. siMAD2L2 + oxaliplatin group; ${ }^{*} \mathrm{P}<0.05$ vs. siMAD2L2 + MG132 group. MAD2L2, mitotic arrest deficient 2 like 2; si, small interfering RNA.

As shown by western blotting, compared with negative control group, siMAD2L2 significantly increased the expression levels of the pro-apoptotic proteins Bax and Bak, as well as decreased the expression level of the anti-apoptotic protein Bcl-2 (Fig. 3). Compared with siMAD2L2 group, cells co-treated with siMAD2L2 and oxaliplatin had increased Bax and Bak expression, and increased Bcl-2 expression. Compared with siMAD2L2 and oxaliplatin co-treatment group, the expression levels of Bax and Bak were significantly decreased after co-treatment with siMAD2L2, oxaliplatin and MG132, while Bcl-2 expression was higher (Fig. 3). This apoptotic effect was further confirmed by the flow cytometry and TUNEL assay results (Figs. 4 and 5). These observations suggested that the Bcl-2 pathway may be involved in the cell apoptosis mediated by MAD2L2.

siMAD2L2-induced suppression of PSMD13 is regulated by oxaliplatin and MG132. Previous studies reported that DNA damage-induced cytotoxic effects were inhibited by MG132 (21-23). Therefore, the activity of PSMD13, a key 
Table III. Activity of PSMD13 in human colorectal cancer cells.

\begin{tabular}{llc}
\hline Treatment & HCT116 & SW620 \\
\hline Negative & $293.21 \pm 31.25$ & $280.31 \pm 6.93$ \\
siMAD2L2 & $385.68 \pm 37.13^{\mathrm{a}}$ & $370.62 \pm 22.43^{\mathrm{a}}$ \\
siMAD2L2 and oxaliplatin & $485.07 \pm 44.17^{\mathrm{a}, \mathrm{b}}$ & $459.15 \pm 28.11^{\mathrm{a}, \mathrm{b}}$ \\
siMAD2L2 and MG132 & $359.72 \pm 36.52^{\mathrm{a}-\mathrm{c}}$ & $334.73 \pm 3.71^{\mathrm{a}-\mathrm{c}}$ \\
siMAD2L2, oxaliplatin and MG132 & $418.46 \pm 33^{\mathrm{a}-\mathrm{d}}$ & $411.28 \pm 2.67^{\mathrm{a}-\mathrm{d}}$
\end{tabular}

${ }^{\mathrm{a}} \mathrm{P}<0.05$ vs. negative group; ${ }^{\mathrm{b}} \mathrm{P}<0.05$ vs. siMAD2L2 group; ${ }^{\mathrm{c}} \mathrm{P}<0.05$ vs. siMAD2L2 + oxaliplatin group; ${ }^{\mathrm{d}}<0.05$ vs. siMAD2L2 $+\mathrm{MG} 132$ group. One-way ANOVA followed by Tukey's multiple comparisons test was used to determine statistical significance. The data are presented as the mean \pm SD. $n=3$. The cells were treated with negative siRNA, siMAD2L2, siMAD2L2 + oxaliplatin, siMAD2L2 + MG132, siMAD2L2 + oxaliplatin + MG132 (oxaliplatin was administered for $24 \mathrm{~h}$ at 50 and $90 \mu \mathrm{M}$ to HCT116 and SW620 cells, respectively; MG132 was administered for $24 \mathrm{~h}$ at 18 and $36 \mu \mathrm{M}$ to HCT116 and SW620 cells, respectively). The activity of PSMD13 was determined using an ELISA. PSMD13, proteasome 26S subunit, non-ATPase 13.

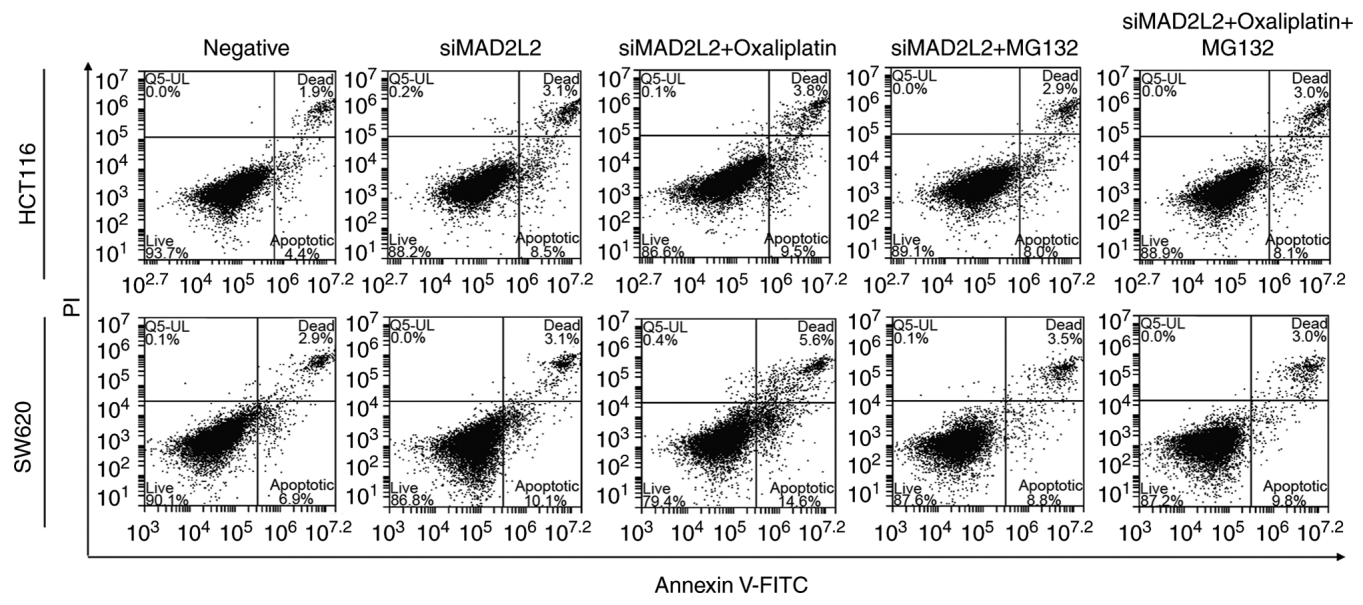

Figure 4. siMAD2L2-induced cell apoptosis is regulated by oxaliplatin and MG132 in HCT116 and SW620 cells. The cells were treated with oxaliplatin or MG132 alone, or co-treated with oxaliplatin and MG132 for $24 \mathrm{~h}$ (oxaliplatin was incubated at 50 and $90 \mu \mathrm{M}$ with HCT116 and SW620 cells, respectively; MG132 was incubated at 18 and $36 \mu \mathrm{M}$ with HCT116 and SW620 cells, respectively). The induction of cell apoptosis was determined via flow cytometry. MAD2L2, mitotic arrest deficient 2 like 2; si, small interfering RNA.

protein of the UPP, was evaluated via ELISA. The results demonstrated that, compared with the negative control group, the activity and expression level of PSMD13 protein were significantly increased in the siMAD2L2 group. Compared with the siMAD2L2 group, PSMD13 activity was mitigated by the co-treatment of siMAD2L2 and MG132, whereas it was promoted by the co-treatment of siMAD2L2 and oxaliplatin. A synergistic effect was not observed during triple co-treatment with siMAD2L2, oxaliplatin and MG132 (Table III), and this effect was further confirmed via western blotting (Fig. 6). These observations indicated that the UPP was involved in the regulation of TLS.

\section{Discussion}

MAD2L2, also called REV7 or MAD2B, encodes a core subunit of DNA polymerase $\zeta$ (24). Previous studies have reported that, in addition to maintaining genomic stability, MAD2L2 was also involved in multiple cellular functions, such as drug resistance reversal, epithelial stromal transformation transcription and signal transduction events (25-27).
In the present study, the aim was to investigate the effects of MAD2L2-induced cell apoptosis.

Previous studies have shown that DNA polymerase $\zeta$ plays an important role in the regulation of platinum resistance and REV3 has been considered the main focus (28); however, the role of MAD2L2 has been underestimated. In the present study, MAD2L2 was selected as the target gene; the results revealed that MAD2L2 promoted oxaliplatin-induced apoptosis. In the present study, human colon cancer cells, HCT116 and SW620, were selected. Cells were characterized for DNA damage by oxaliplatin. The protein expression level of MAD2L2 was found to be significantly downregulated. Flow cytometry and TUNEL results demonstrated that treatment with siMAD2L2 or oxaliplatin alone increased the apoptosis of both HCT116 and SW620 cells, whereas cells co-treated with siMAD2L2 and oxaliplatin significantly promoted apoptosis. The results of western blotting showed that knockdown of MAD2L2 expression caused by RNA interference or oxaliplatin increased the expression levels of pro-apoptotic proteins Bax and Bak and decreased the expression levels of anti-apoptotic protein $\mathrm{Bcl}-2$, compared with the negative control group. These results 


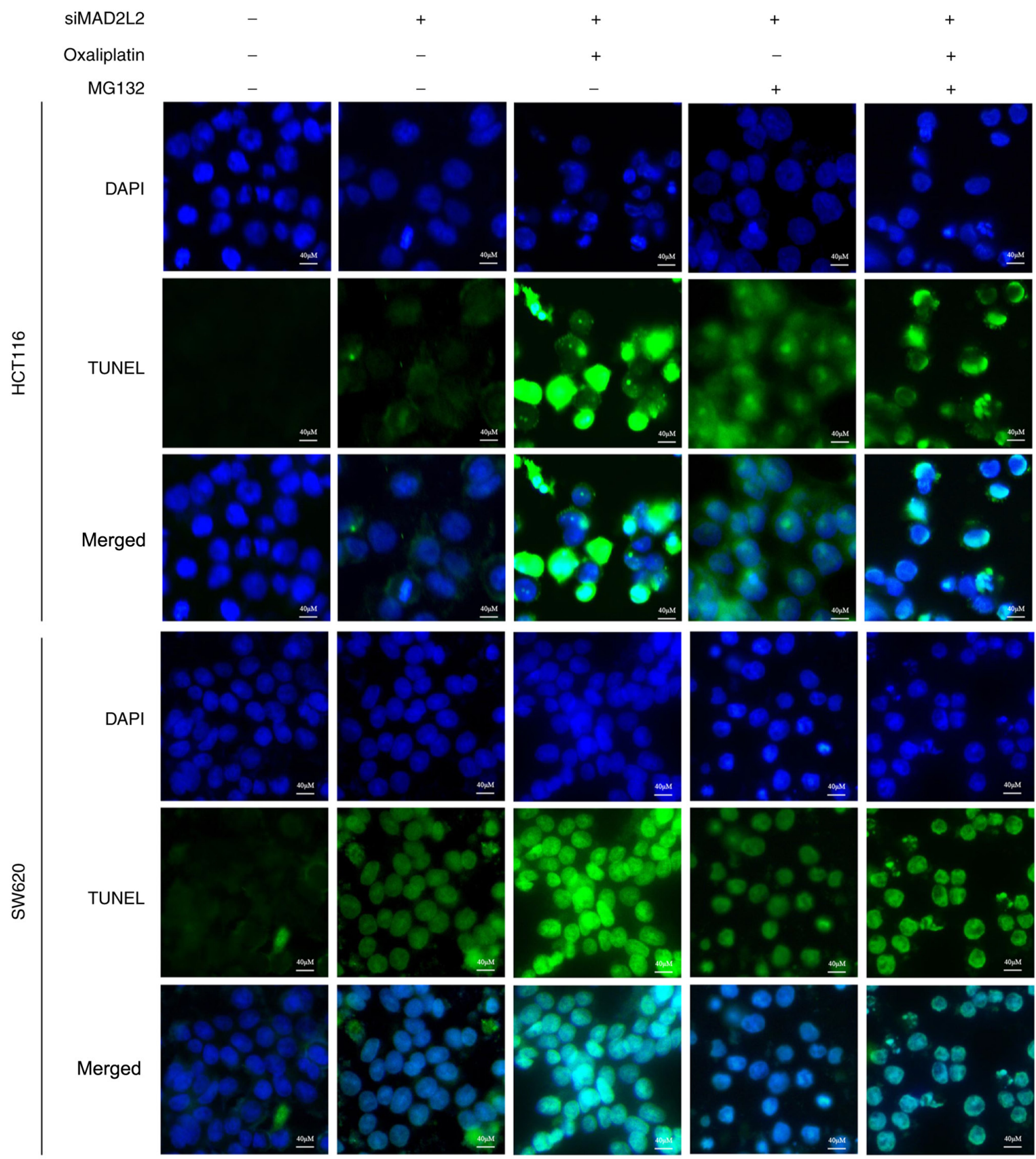

Figure 5. siMAD2L2-induced cell apoptosis is regulated by oxaliplatin and MG132 in HCT116 and SW620 cells. The cells were treated with oxaliplatin or MG132 alone, or co-treated with oxaliplatin and MG132 for $24 \mathrm{~h}$ (oxaliplatin was incubated at 50 and $90 \mu \mathrm{M}$ with HCT116 and SW620 cells, respectively; MG132 was incubated at 18 and $36 \mu \mathrm{M}$ with HCT116 and SW620 cells, respectively). The induction of cell apoptosis was determined using TUNEL staining. Scale bar, $40 \mu \mathrm{m}$. MAD2L2, mitotic arrest deficient 2 like 2; si, small interfering RNA.

indicated that oxaliplatin promoted siMAD2L2-induced apoptosis of colon cancer cells, and this process was associated with the Bcl-2 family mediated cell apoptosis pathway.

In order to investigate the causes of MAD2L2-induced cell apoptosis, the present study focused on the UPP, which is responsible for the majority of intracellular protein degradation (29). This system exerts its biological effect via the cooperation of $\mathrm{E} 1 / \mathrm{E} 2 / \mathrm{E} 3$. Ub is activated by E1 and is then transferred to E2, which permits it to be sequentially conjugated to E3. E3 recognizes target substrates and catalyzes the covalent attachment of $\mathrm{Ub}$ to it (30). Finally, the substrates modified with polyubiquitin chains are delivered to the $26 \mathrm{~S}$ proteasome for proteolytic destruction. Here, PSMD13 serves an important role (31).
In the present study, the activity of PSMD13 was evaluated via ELISA and further confirmed by western blotting. The results showed that the activity and protein expression level of PSMD13 were significantly increased by siMAD2L2. In addition, MG132, the inhibitor of proteasome, decreased the expression of MAD2L2, while reducing the siMAD2L2-induced cell apoptosis. These results suggest that the UPP was implicated in the regulation of TLS.

TLS depends on the orderly assembly of DNA polymerases (32). Cells are constantly exposed to DNA damage agents, such as UV, methyl methanesulfonate and other cytotoxic factors (33). Once DNA damageoccurs, proliferating cellnuclear antigen (PCNA) can be recruited and mono-ubiquitinated 
A

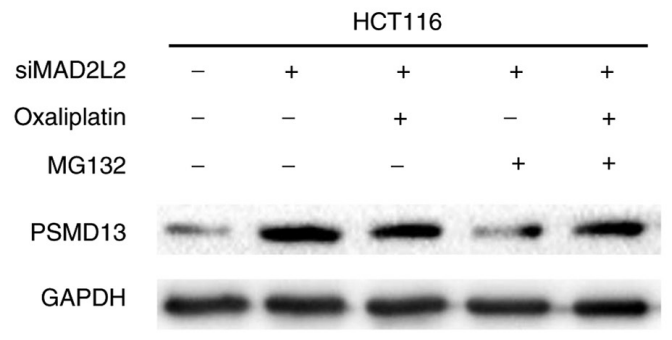

B

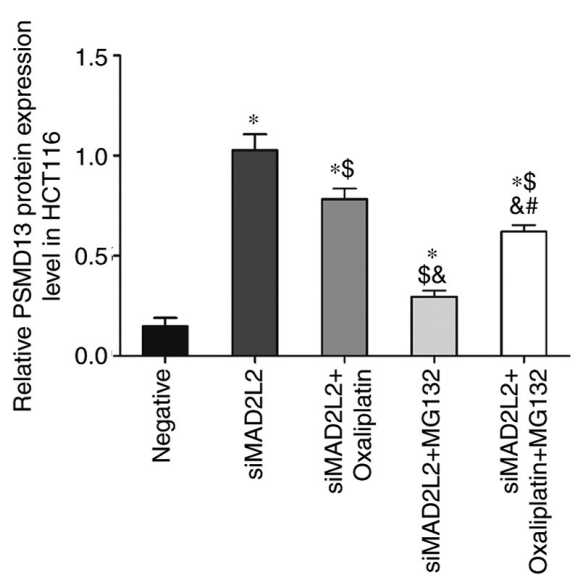

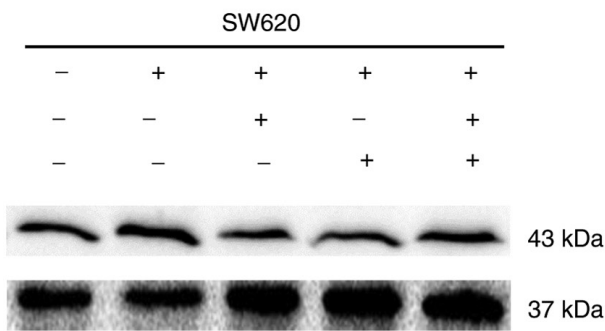

$\mathrm{C}$

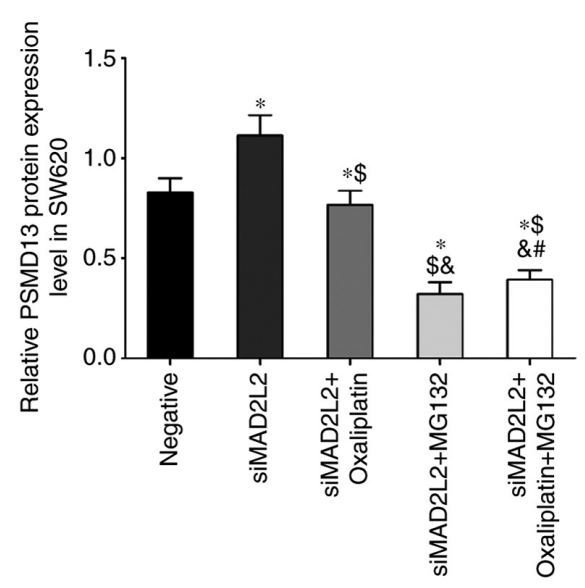

Figure 6. siMAD2L2-induced suppression of PSMD13 is regulated by oxaliplatin and MG132. The cells were treated with negative siRNA, siMAD2L2, siMAD2L2 + oxaliplatin, siMAD2L2 + MG132, siMAD2L2 + oxaliplatin + MG132 (oxaliplatin was incubated for $24 \mathrm{~h}$ at 50 and $90 \mu \mathrm{M}$ with HCT116 and SW620 cells, respectively; MG132 was incubated for $24 \mathrm{~h}$ at 18 and $36 \mu \mathrm{M}$ with HCT116 and SW620 cells, respectively). (A) Western blotting was performed to detect the protein levels of PSMD13 in HCT116 and SW620 cells. One-way ANOVA followed by Tukey's multiple comparisons test was used to determine the statistical significance in (B) HCT116 and (C) SW620 cells. The data are presented as the mean \pm SD. n=3. ${ }^{*} \mathrm{P}<0.05$ vs. negative group; ${ }^{\$} \mathrm{P}<0.05$ vs. siMAD2L2 group; ${ }^{\circledR} \mathrm{P}<0.05$ vs. siMAD2L2 + oxaliplatin group; ${ }^{\mathrm{P}} \mathrm{P} 0.05$ vs. siMAD2L2 + MG132 group. MAD2L2, mitotic arrest deficient 2 like 2; si, small interfering RNA; PSMD13, proteasome 26S subunit, non-ATPase 13.

by Rad18-Rad6 at Lys164 (K164), and Ub-PCNA mediates cellular response by TLS polymerases $(34,35)$. MAD2L2 helps to coordinate the nucleotide insertion and extension steps of lesion bypass (36). Interestingly, $\operatorname{Rad} 18$ and Rad6 are particularly important E2 and E3 enzymes, which function as Ub conjugating and Ub ligase enzymes, respectively, and have been shown to be involved in the UPP, which mediates the degradation of proteins (37).

In the present study, the protein expression level of MAD2L2 was inhibited when the cells were treated with MG132. In addition, cell apoptosis was reduced by MG132 compared with the co-treatment of siMAD2L2 and oxaliplatin. Based on these results, it was suggested that the UPP may be one of the responders of TLS-related DNA damage in colon cancer cells. DNA lesions that are induced by oxaliplatin or siMAD2L2 may promote the cooperation between Rad6 and Rad18, which then activate PSMD13, finally resulting in the degradation of MAD2L2 protein. Such agents arrest TLS, trigger the accumulation of DNA damage and promote cancer cell apoptosis (38).

Although the present study indicated that both oxaliplatin and MG132 exerted impacts on siMAD2L2-induced cell apoptosis, these factors were not simply synergistic or antagonistic. More direct evidence is needed for effective treatment of colon cancer in the future. In conclusion, the present study demonstrated oxaliplatin promoted siMAD2L2-induced colon cell apoptosis, which was regulated by the UPP. Overall, the present study provides a theoretical basis for improving the clinical efficacy of colon cancer by combining chemotherapy and gene therapy.

\section{Acknowledgements}

I would like to extend my sincere gratitude to Professor Hao Wang (School of Pharmacy, Minzu University of China), Professor Jianmin Sun (School of Basic Medical Sciences, Ningxia Medical University) for their intellectual guidance, valuable comments and enlightening suggestions for my study and manuscript preparation.

\section{Funding}

The present study was supported by the National Natural Science Foundation of China (grant no. 31360251) and West China first-class Disciplines Basic Medical Sciences at Ningxia Medical University (grant no. NXYLXK2017B07).

\section{Availability of data and materials}

The datasets used during the current study are available from the corresponding author on reasonable request.

\section{Authors' contributions}

LM and FX designed the study. YL performed cell culture, transfection experiments and drug sensitivity tests. HTS contributed to the ELISA and western blotting assays. XPZ and XL performed the flow cytometry and TUNEL assays. LM and HTS acquired and interpretated the data. FFK and YS assisted 
with data analysis. LM, XL and YL drafted the manuscript. HTS and LM are responsible for confirming the authenticity of the raw data. All authors read and approved the final manuscript.

\section{Ethics approval and consent to participate}

Not applicable.

\section{Patient consent for publication}

Not applicable.

\section{Competing interests}

The authors declare that they have no competing interests.

\section{References}

1. Siegel RL, Miller KD and Jemal A: Cancer statistics, 2020. CA Cancer J Clin 70: 7-30, 2020.

2. Crowley LC, Marfell BJ and Waterhouse NJ: Detection of DNA fragmentation in apoptotic cells by TUNEL. Cold Spring Harb Protoc: 2016, 2016.

3. Zhou J, Zheng R, Zhang S, Zeng H, Wang S, Chen R, Sun K, $\mathrm{Li}$ M, Gu J, Zhuang G and Wei W: Colorectal cancer burden and trends: Comparison between China and major burden countries in the world. Chin J Cancer Res 33: 1-10, 2021.

4. National Health Commission Of The People's Republic Of China: National guidelines for diagnosis and treatment of colorectal cancer 2020 in China (English version). Chin J Cancer Res 32: 415-445, 2020.

5. Sharma G, Anghore D, Khare R and Rawal RK: Oxaliplatin for colorectal cancer therapy: A review. Clin Cancer Drugs 5: 13-27, 2018.

6. Brown A, Kumar S and Tchounwou PB: Cisplatin-based chemotherapy of human cancers. J Cancer Sci Ther 11: 97, 2019.

7. McQuade RM, Stojanovska V, Bornstein JC and Nurgali K Colorectal cancer chemotherapy: The evolution of treatment and new approaches. Curr Med Chemistry 24: 1537-1557, 2017.

8. Sigel A, Riddell IA, Lippard SJ, Sigel H, Freisinger E and Sigel RKO: Cisplatin and oxaliplatin: Our current understanding of their actions. 1-42, 2018.

9. Ma X, Tang TS and Guo C: Regulation of translesion DNA synthesis in mammalian cells. Environ Mol Mutagen 61: 680-692, 2020

10. Shanbhag V, Sachdev S, Flores JA, Modak MJ and Singh K: Family A and B DNA polymerases in cancer: Opportunities for therapeutic interventions. Biology (Basel) 7: 5, 2018.

11. Sharma S, Shah NA, Joiner AM, Roberts KH and Canman CE: DNA polymerase zeta is a major determinant of resistance to platinum-based chemotherapeutic agents. Mol Pharmacol 81: 778-787, 2012.

12. Jiang HG, Chen P, Su JY, Wu M, Qian H, Wang Y and Li J: Knockdown of REV3 synergizes with ATR inhibition to promote apoptosis induced by cisplatin in lung cancer cells. J Cell Physiol 232: 3433-3443, 2017.

13. Malik R, Kopylov M, Gomez-Llorente Y, Jain R, Johnson RE, Prakash L, Prakash S, Ubarretxena-Belandia I and Aggarwal AK: Structure and mechanism of B-family DNA polymerase zeta specialized for translesion DNA synthesis. Nat Struct Mol Biol 27: 913-924, 2020.

14. Washington MT and Gildenberg MS: Structure of DNA polymerase $\xi$ : Capturing the getaway driver. Nat Struct Mol Biol 27: 1-2, 2020.

15. Suzuki T, Sassa A, Gruz P, Gupta RC, Johnson F, Adachi N and Nohmi T: Error-prone bypass patch by a low-fidelity variant of DNA polymerase zeta in human cells. DNA Repair (Amst) 100: $103052,2021$.

16. Gaczynska M and Osmulski PA: Targeting protein-protein interactions in the ubiquitin-proteasome pathway. Adv Protein Chem Struct Biol 110: 123-165, 2018

17. Bi W, Zhu L, Zeng Z, Jing X, Liang Y, Guo L, Shi Q, Xu A and Tao E: Investigations into the role of $26 \mathrm{~S}$ proteasome non-ATPase regulatory subunit 13 in neuroinflammation. Neuroimmunomodulation 21: 331-337, 2014.
18. Bard JAM, Goodall EA, Greene ER, Jonsson E, Dong KC and Martin A: Structure and function of the 26S proteasome. Ann Rev Biochem 87: 697-724, 2018.

19. Chen X, Htet ZM, Lopez-Alfonzo E, Martin A and Walters KJ: Proteasome interaction with ubiquitinated substrates: From mechanisms to therapies. FEBS J: Nov 19, 2020 (Epub ahead of print).

20. Livak KJ and Schmittgen TD: Analysis of relative gene expression data using real-time quantitative PCR and the 2(-Delta Delta C(T)) method. Methods 25: 402-408, 2001

21. Tu Y, Chen C, Pan J, Xu J, Zhou ZG and Wang CY: The Ubiquitin Proteasome Pathway (UPP) in the regulation of cell cycle control and DNA damage repair and its implication in tumorigenesis. Int J Clin Exp Pathol 5: 726-738, 2012.

22. Sakai W, Yuasa-Sunagawa M, Kusakabe M, Kishimoto A, Matsui T, Kaneko Y, Akagi JI, Huyghe N, Ikura M, Ikura T, et al: Functional impacts of the ubiquitin-proteasome system on DNA damage recognition in global genome nucleotide excision repair. Sci Rep 10: 19704, 2020.

23. Ramadan K and Meerang M: Degradation-linked ubiquitin signal and proteasome are integral components of DNA double strand break repair: New perspectives for anti-cancer therapy. FEBS Lett 585: 2868-2875, 2011.

24. Rizzo AA, Vassel FM, Chatterjee N, D'Souza S, Li Y, Hao B, Hemann MT, Walker GC and Korzhnev DM: Rev7 dimerization is important for assembly and function of the Rev1/Pols translesion synthesis complex. Proc Natl Acad Sci USA 115: E8191-E8200, 2018.

25. Feng L, Wei W, Heng Z, Yantao H and Chunbo W: Knockdown of REV7 inhibits breast cancer cell migration and invasion. Oncol Res 24: 315-325, 2016.

26. Rosenberg SC and Corbett KD: The multifaceted roles of the HORMA domain in cellular signaling. J Cell Biol 211: 745-755, 2015.

27. Hara K, Taharazako S, Ikeda M, Fujita H, Mikami Y, Kikuchi S, Hishiki A, Yokoyama H, Ishikawa Y, Kanno SI, et al: Dynamic feature of mitotic arrest deficient 2-like protein 2 (MAD2L2) and structural basis for its interaction with chromosome alignment-maintaining phosphoprotein (CAMP). J Biol Chem 292: 17658-17667, 2017

28. Sanders MA, Haynes B, Nangia-Makker P, Polin LA and Shekhar MP: Pharmacological targeting of RAD6 enzyme-mediated translesion synthesis overcomes resistance to platinum-based drugs. J Biol Chemistry 292: 10347-10363, 2017.

29. Jantrapirom S, Piccolo LL, Pruksakorn D, Potikanond S and Nimlamool W: Ubiquilin networking in cancers. Cancers 12: 1206-1586, 2020.

30. Leestemaker $\mathrm{Y}$ and Ovaa $\mathrm{H}$ : Tools to investigate the ubiquitin proteasome system. Drug discovery today. Technologies 26: 25-31, 2017

31. Wehmer M, Rudack T, Beck F, Aufderheide A,Pfeifer G, Plitzko JM, Förster F, Schulten K, Baumeister W and Sakata E: Structural insights into the functional cycle of the ATPase module of the 26S proteasome. Proc Natl Acad Sci USA 114: 1305-1310, 2017.

32. Rizzo AA and Korzhnev DM: The Rev1-Pol' translesion synthesis mutasome: Structure, interactions and inhibition. Enzymes 45: 139-181, 2019.

33. Fernandes E, Fonseca TG, Carriço T, Mestre N, Tavares Á and Bebianno MJ: Cytotoxic responses of the anticancer drug cyclophosphamide in the mussel Mytilus galloprovincialis and comparative sensitivity with human cells lines. Chemosphere 261: 127678, 2020.

34. Kanao R and Masutani C: Regulation of DNA damage tolerance in mammalian cells by post-translational modifications of PCNA. Mutat Res 803-805: 82-88, 2017.

35. Lau WCY,Li Y,Zhang Q and Huen MSY: Molecular architecture of the Ub-PCNA/Pol $\eta$ complex bound to DNA. Sci Rep 5: 15759, 2015.

36. Quinet A, Lerner LK, Martins DJ and Menck CFM: Filling gaps in translesion DNA synthesis in human cells. Mutat Res Genet Toxicol Environ Mutagen 836: 127-142, 2018

37. An H, Yang L, Wang C, Gan Z, Gu H, Zhang T, Huang X, Liu Y, $\mathrm{Li}$ Y, Chang SJ, et al: Interactome analysis reveals a novel role for RAD6 in the regulation of proteasome activity and localization in response to DNA damage. Mol Cell Biol 37: e00419-e00416, 2017.

38. Masuda Y and Masutani C: Spatiotemporal regulation of PCNA ubiquitination in damage tolerance pathways. Crit Rev Biochem Mol Biol 54: 418-442, 2019.

This work is licensed under a Creative Commons Attribution-NonCommercial-NoDerivatives 4.0 International (CC BY-NC-ND 4.0) License. 